\title{
Are we ready for the Digital Age?
}

\author{
Daniel P. Raymond, MD
}

\author{
From Thoracic Surgery, Cleveland Clinic Health System, Cleveland, Ohio. \\ Disclosures: Author has nothing to disclose with regard to commercial support. \\ Received for publication Oct 12, 2017; accepted for publication Oct 13, 2017. \\ Address for reprints: Daniel P. Raymond, MD, Thoracic Surgery, Cleveland Clinic Health System, 9500 Euclid \\ Ave, J4-1, Cleveland, OH 44195) (E-mail: raymond3@ccf.org). \\ J Thorac Cardiovasc Surg 2018; 155:1841-2 \\ $0022-5223 / \$ 36.00$ \\ Copyright (c) 2017 by The American Association for Thoracic Surgery \\ https://doi.org/10.1016/j.jtcvs.2017.10.043
}

"The nurse recorded a leak this morning, but I don't see one. The Nurse Practitioner thinks it's a swing and not a leak." Who has not experienced this ritual in the morning? The collation of opinions from morning rounds ultimately lead to the decision to leave it, clamp it, or pull it. In the era ruled by quality and cost, these decisions need to be made with increasing accuracy and alacrity to reduce length of stay while starving our Society of Thoracic Surgeons data collectors of the undesirable check in the Postoperative Events section of their data-collection form. An objective, digital pleural drainage device seems a logical answer to this challenge. To quote a senior thoracic surgeon, "Any device that objectifies an inherently subjective patient measurement is going to be better."' Enter the Thopaz pleural drainage system (Medela, Baar, Switzerland).

Dr Takamochi and colleagues ${ }^{2}$ at the Jutendo University School of Medicine have added to the growing literature on the use of digital pleural drainage devices for the management of patients after lung resection. They compare a digital drainage system with a traditional drainage system and designate the primary end point as chest tube duration, which avoids the complex, multifactorial debate related to length of stay end points. They determine a 1-day reduction in chest tube duration as clinically relevant and are able to achieve that difference in the study, a median of 2.0 days versus 3.0 days, but are not able to reach statistical significance $(P=.149)$. The study is complicated by a preoperative randomization method that contributes to a significantly higher leak rate in one group, thus introducing bias. Additional concerns arise in the postoperative management because there is a 6-fold higher rate of pleurodesis in the digital drainage group. The authors ultimately conclude that they did not identify a shortened chest tube duration; however, their Perspective statement proposing a "well-planned prospective randomized study"2 suggests their enthusiasm for this technology has not deflated.

Presently, studies exist in the literature falling on both sides of the fence regarding the utility of electronic pleural drainage systems. A notable positive study was the multicenter, international randomized trial by Pompili and colleagues, ${ }^{3}$ which demonstrated a 1-day reduction in chest tube duration

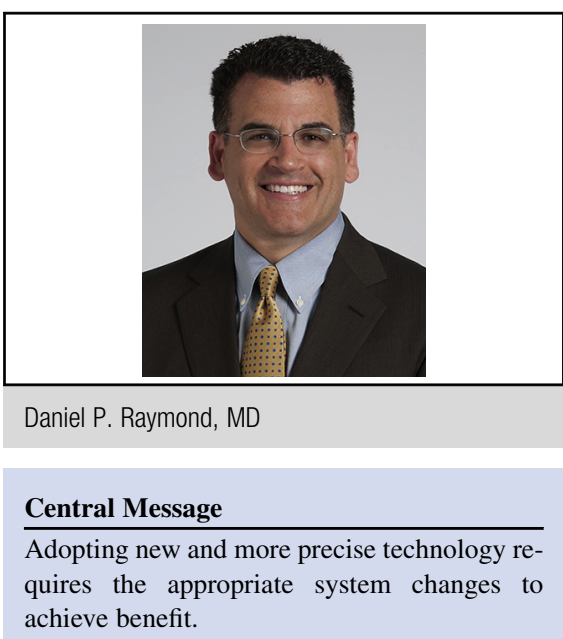

See Article page 1834. (1.0 vs 2.2 days; $P=.001$ ) and a 1-day reduction in postoperative length of stay (4.6 vs 5.6 days, $P<.001$ ). However, a challenge of this increased objectivity and precision is the need for a significant cultural change in thoracic surgery. Current culture commonly involves chest tube management decisions in 24-hour increments. This is generally based on the desire to remove tubes early in the day to have "all hands on deck" should difficulties arise. Furthermore, this permits appropriate time for preparation of the patient for discharge. This practice is practical, safe, and, admittedly, convenient for practicing surgeons who want to minimize late trips back to the hospital. In the randomized study by Lijkendijk and colleagues ${ }^{4}$ they noted, "We also discovered that many of the chest tubes were removed in close proximity to morning rounds, which could reflect staff nurses were not comfortable making the decision to remove a chest tube despite our algorithm or that it was simply daytime when most staff were present." Simply put, if we are going to take advantage of the precision that these devices can potentially provide, we must create systems that accommodate that precision. A fast-track pathway that includes chest tube removal at the time the definition of "no air leak" has been achieved is plausible but would require a concerted multidisciplinary effort, as well as the ability to correct problems at inconvenient times without compromise to patient safety.

An additional challenge of these devices is cost neutrality. Brunelli and colleagues ${ }^{5}$ demonstrated a statistically significant reduction in chest tube duration and length of stay, as well as a $€ 476$ cost reduction per patient. ${ }^{5}$ This savings is likely highly dependent on 
center-dependent performance variables, such as current length of stay, air leak rate after resection, and contractdetermined material costs that would influence the degree of savings. Narrowing the application of this technology to higher-risk/high-cost scenarios (eg, leak identified at end of case, redo surgery) may be a wise practice to increase the potential benefit. Finally, there are other potential benefits, including patient satisfaction, mobility, and the potential to shorten the duration of an air leak that warrant consideration.

I would agree with the senior thoracic surgeon quoted previously $^{1}$ that this technology is likely a step in the right direction for thoracic surgery. Unfortunately, however, I do not think appropriate use of it is as simple as opening the box.

\section{References}

1. Gilbert S, McGuire AL, Sonam M, Sundaresen SR, Seely AJ, Maziak DE, et al. Randomized trial of digital versus analog pleural drainage in patients with or without a pulmonary air leak after lung resection. J Thorac Cardiovasc Surg. 2015;150:1243-51

2. Takamochi K, Nojiri S, Oh S, Matsunaga T, Imashimizu K, Fukui M, et al. Comparison of a digital with a traditional drainage system for the postoperative chest tube management after pulmonary resection: a prospective randomized trial. J Thorac Cardiovasc Surg. 2018;155:1834-40.

3. Pompili C, Detterbeck F, Papagiannopoulos K, Sihoe A, Vashlas K, Maxfield MW, et al. Multicenter international randomized comparison of objective and subjective outcomes between electronic and traditional chest drainage systems. Ann Thorac Surg. 2014;98:490-7.

4. Lijkendijk M, Licht PB, Neckelmann K. Electronic versus traditional chest tube drainage following lobectomy: a randomized trial. Eur J Cardiothorac Surg. 2015;48:893-8.

5. Brunelli B, Salati M, Refai M, Di Nunzio L, Xiume F, Sabbatini A. Evaluation of a new chest tube removal protocol using digital air leak monitoring after lobectomy: a prospective trial. Eur J Cardiothorac Surg. 2010;37:56-60. 